usually 2 or 3 times higher than the general population. The prevalence of $1 / 45$ quoted by the present Turkish group was not statistically significant [1]. In one large-scale study, the frequency of CD confirmed by the antiendomysial antibody IgA scan and small intestine biopsy was $1 / 127$ in epileptic patients $(n=255)$ and $1 / 293$ in a control group $(n=3,400)[2]$.

\title{
References.
}

1. Dai AI, et al. Childs Nerv Syst. 2014 Jun;30(6):1091-8.

2. Pratesi R, et al. Arq Neuropsiquiatr. 2003 Jun;61(2B):330-4.

\section{EPILEPSY AS AN AUTOIMMUNE DISEASE}

Investigators at University of New South Wales, Sydney, Australia, and Boston Children's Hospital, Harvard Medical School, conducted a retrospective population-level study of the relationship between epilepsy and 12 common autoimmune diseases: type 1 diabetes mellitus, psoriasis, rheumatoid arthritis, Graves disease, Hashimoto thyroiditis, Crohn disease, ulcerative colitis, systemic lupus erythematosus, antiphospholipid syndrome, Sjogren syndrome, myasthenia gravis, and celiac disease. The risk of epilepsy was significantly heightened among patients with all autoimmune diseases $(\mathrm{P}<0.001)$, and especially in children. Children with autoimmune diseases had a 5-fold increased risk of epilepsy. (Ong M-S, Kohane IS, Cai T, Gorman MP, Mandl KD. Population-level evidence for an autoimmune etiology of epilepsy. JAMA Neurol 2014 May;71(5):56974).

COMMENTARY. The authors theorize that the occurrence of epilepsy in patients with autoimmune disease (AD) might be attributable to the inflammatory component of AD [1]. Also, certain anticonvulsant drugs, such as valproate and carbamazepine, are anti-inflammatory, and some anti-inflammatory drugs have anticonvulsant properties [2].

Investigators at the Lurie Children's Hospital of Chicago have provided several reports of the role of brain inflammation in epileptogenesis. Cortical tissue collected from children with intractable epilepsy at time of surgery showed active neuroinflammation and marked cellular injury [3]. In a review of brain inflammation in the pathogenesis of epilepsy, common pediatric autoimmune diseases are implicated, and the effective use of anti-inflammatory treatments of intractable epilepsy, such as steroids, is documented [4].

\section{References.}

1. Ong M-S, et al. JAMA Neurol. 2014 May;71(5):569-74.

2. Marchi N, et al. PLoS One. 2011 Mar 28;6(3):e18200.

3. Choi J, Nordli DR Jr, Alden TD, et al. J Neuroinflammation. 2009 Dec 19;6:38.

4. Choi J, Koh S. Yonsei Med J. 2008 Feb 29;49(1):1-18.

\section{MAINTENANCE THERAPY FOR MYASTHENIA GRAVIS}

Investigators at Boston Children's Hospital and at University of Florida, Gainesville, studied the comparative efficacy of plasmapheresis (PLEX) vs immunoglobulin (IVIG) as maintenance therapy in juvenile myasthenia gravis (MG). A retrospective analysis over a 33-year period involved 54 children and adolescents with 